\title{
EL POBLAMIENTO RURAL EN EL ARROYO DE GUADATIN: UN MODELO DE OCUPACION DEL TERRITORIO DURANTE EL BRONCE FINAL Y EL PERIODO ORIENTALIZANTE EN EL VALLE MEDIO DEL GUADALQUIVIR
}

\author{
Juan F. MURILLO REDONDO (*) \\ José A. MORENA LOPEZ (**)
}

\section{Resumen}

Este trabajo ofrece un modelo de ocupación del territorio en el Valle Medio del Guadalquivir a partir del análisis de medio centenar de pequeños asentamientos rurales encuadrables en los momentos finales de la Edad del Bronce y en el Orientalizante. Se abordan las estrechas relaciones entre el medio físico y las estrategias de explotación agrícola, así como los mecanismos de distribución de productos manufacturados, especialmente cerámicas, en el medio rural.

\section{Summary}

This paper tries to explain the territorial pattem of around fifty farms located in the Middle Valley of Guadalquivir river during the Late Bronze Age and the Orientalizing Period. The close relationships between landscape and agriculture are widely studied and also the distribution of manufactured goods, including pottery, in the rural environment.

El Arroyo Guadatín es uno de los muchos afluentes que, dentro de su tramo medio, recibe el Guadalquivir por la izquierda, formando una red fluvial que drena un pequeño sector de la Campiña de Córdoba. Con una longitud de más de una veintena de kilómetros y una anchura que oscila entre los 250 y $\operatorname{los} 1000 \mathrm{~m}$., queda configurado un valle fluvial que ofrece grandes atractivos para la ocupación humana.

(*) Seminario de Arqueología. Universidad de Córdoba.

(**) Museo Histórico Local. Cañete de las Torres, Córdoba. 
Una serie de prospecciones sistemáticas efectuadas a lo largo de este curso fluvial han permitido la localización de un total de cuarenta y cuatro pequeños asentamientos encuadrables, a partir de los materiales arqueologicos recogidos en superficie, en la fase Orientalizante del Bronce Final. El horizonte cultural que estos yacimientos nos proporcionan es relativamente homogéneo, con cerámicas fabricas a mano entre las que destacan cazuelas bruñidas de perfil evolucionado y, en una proporción muy superior, vasos de tendencia globular, base plana y superficies toscas. Las decoraciones se limitan a algún fragmento de retícula bruñida y a incisiones, impresiones de punzón, digitaciones y mamelones de distintos tipos. Las cerámicas fabricadas a torno se reducen a varias formas de pasta gris y superficies bruñidas o alisadas, y a decoraciones de anchas bandas y ćrculos concéntricos pintados con bicromfa de rojos y negros, a menudo sobre un engobe de color crema. Es significativa la ausencia de engobe rojo y de decoraciones figuradas de tipo orientalizante.

Todo esto nos da un horizonte cronólogico centrado grosso modo desde inicios del siglo VII hasta mediados del s. VI. Por otro lado, la evaluación de los recursos potenciales de la zona (suelos y aprovisionamiento de agua) junto a diversos vestigios extraidos del contexto arqueológico (molinos de mano, denticulados, dientes de hoz), demuestran la orientación agrícola, más concretamente cerealista de estas explotaciones rurales.

La afortunada coincidencia de todos estos factores, espacio geográfico homogéneo, intervalo cronológico circunscribible a algo más de siglo y medio, y una sorprendente alta densidad de asentamientos, nos ha llevado a realizar una análisis locacional tendente a definir la correlación existente entre los asentamientos y las variables consideradas, asf como el patrón de asentamiento y su integración dentro de la red primaria de poblamiento establecido para la Cuenca Media del Guadalquivir (MURILLO, 1991).

Como ya expusimos más arriba, el Arroyo Guadatín pone en contacto la Campiña con el Valle del Guadalquivir, constituyendo una de las escasas vías naturales de penetración al interior de aquélla, hacia las tierras de Bujalance. Cañete de las Torres y Castro del Rfo. Desde el punto de vista geológico podemos distinguir dos sectores, uno, el mayoritario, dominio de los terrenos miocenos campiñeses, y otro, circunscrito a los últimos kms. de su curso, ya en tierras del actual término municipal de Villafranca, constituido por materiales cuaternarios de origen aluvial.

Esta diferenciación tiene un gran interés edafológico, pues mientras que el sector mioceno presenta los típicos suelos margosos béticos, el cuatemario muestra suelos rojos y pardorrojizos mediterráneos sobre sedimentos aluviales (CEBAC, 1971). También debemos tener presente la existencia de pequeñas manchas de tierras negras andaluzas sobre margas, como en la confluencia del Arroyo de Lorilla con el Guadatín. De acuerdo con la jerarquización que hemos establecido para los suelos de la provincia de Córdoba 
(MURILLO, 1991) a partir de su potencialidad para aprovechamientos agrícolas, ganaderos y forestales, todos estos suelos se incluirian dentro de la Categoría B (LaborAlta productividad-Secano), especialmente apta para el cultivo de cereales.

Por lo que respecta a los recursos hídricos, su aprovisionamiento supone un claro problema en la Campiña, y de forma más concreta en su reborde septentrional. El agua es un factor al que se ha concedido escasa atención en los estudios de nuestra Prehistoria reciente y Protohistoria, salvo en su relación con la práctica de una agricultura de regadío (GILMAN et alii, 1985). Y, sin embargo, es por todos aceptado su carácter condicionante de la instalación estable de un grupo humano. La presencia de manantiales suele representar una poderosa atracción para la ocupación humana, que se perpetúa en tomo a los mismos durante generaciones. No obstante, en zonas donde la geología no es proclive a que el agua mane de forma natural, los cauces fluviales constiuyen los únicos puntos de aprovisionamiento directo de agua.

Para el caso concreto del Guadatín, de los 44 asentamientos contabilizados, la distancia mínima al arroyo es de una decena de metros, en tanto que la máxima es de 350. La distancia media se sitúa en los $90 \mathrm{~m}$. Así pues, el aprovechamiento de las aguas del arroyo para el consumo humano sería factible en la relación esfuerzo invertido/volumen de recurso apropiado. Pero la ya señalada naturaleza geológica del terreno, unida al régimen hidrológico de los cursos fluviales de la Campiña, caracterizados por unas rápidas crecidas originadas por una corta estación de lluvias que suelen adquirir un carácter torrencial, y por un acusado estiaje favorecido por la permeabilidad del terreno y por la alta evaporación, hacen que sólo en muy contados momentos estacionales estas aguas fueran aprovechables, salvo que se emplearan sofisticados sistema de regulación y decantación-filtración de las mismas.

$\mathrm{La}$ ausencia de capas impermeables que permitan la existencia de manantiales impide un aprovechamiento inmediato y directo de las aguas subterráneas, obligando a la perforación de pozos. De acuerdo con el Mapa Hidroclimático de Andalucía (ROSA et MOREIRA, 1987) el área que venimos estudiando cuenta con importantes recursos acufferos, estimados en los 100-200 hectómetros cúbicos anuales, de origen detrítico y facies clorurada. Una moderada salinidad permite el consumo humano de estas aguas. El nivel freático se halla por encima de la isopieza de los $100 \mathrm{~m}$., siendo lo normal el encontrar agua a partir de los $10620 \mathrm{~m}$. de profundidad. En consecuencia, nos queda ésta como la única posibilidad de asegurar un aprovisionamiento estable de agua. La peculiaridad de este tipo de estructura hace muy difícil su documentación arqueológica y mucho más su datación cronológica, lo que explica que no podamos presentar ningún ejemplo de pozo fechable en las etapas que aquí nos interesan. No obstante, diversas construcciones localizadas en el poblado de Valencina de la Concepción, con una amplia ocupación que abarca desde el Calcolítico al Bronce Final, han sido interpretadas como sistemas de captación de aguas subterráneas. 
Como una primera aproximación espacial al poblamiento en el Arroyo Guadatín, hemos aplicado la técnica del Vecino más Próximo (Nearest Neighbour Analysis). se trata de un método probabilítico y estadístico basado en la medición de la distancia real, en línea recta, entre un punto y su vecino más próximo (Fig. 2), sin tener en cuenta las posibles características distintas de cstos, y en la comparación entre estas distancias y las que se hallarían teóricamente si los puntos se hallasen distribuidos de modo aleatorio en el interior de la misma superifie (HAGGETT, 1976). La comparación se efectúa mediante el índice del Vecino más Próximo $(\mathrm{R} n)$, obtenido de la fórmula:

\section{$\mathrm{R} \mathrm{n}=2 \times \mathrm{d} \times(\mathrm{N} / \mathrm{A})$}

donde $d$ es la distancia media real entre los puntos de la distribución, $N$ es el número de puntos considerados, y $A$ la superficie que contiene los puntos. Esta última constituye la variable más problemática, pues obliga a fijar unos límites que no siempre aparecen claros. De este modo, dependiendo de la escala tomada (HODDER et OKELL, 1978), se pueden generar distorsiones, de las cuales la más importante es la conocida como efecto limite (ORTON, 1988).

No hemos enfrentado a este problema partiendo de la clara simetría que introduce el arroyo Guadatín en cuanto eje longitudinal sobre el que se articula el poblamiento. Tomando en consideración la distancia máxima entre un punto y el Arroyo (750 m. para el yacimiento $n^{\circ} 120$ ) obtendríamos ya una anchura de $1500 \mathrm{~m}$. para la franja de terreno en estudio. Como de todos modos el asentamiento 120 precisaría de un territorio de explotación a su alrededor, hemos ampliado esa anchura en otros $500 \mathrm{~m}$. (250 m. a cada lado del eje marcado por el Guadatín). Esta ampliación, equivalente a dos tercios de la distancia media entre los puntos de la distribución, nos da una anchura total de $2000 \mathrm{~m}$., que viene a coincidir con el límite impuesto al valle fluvial en sus tramos alto y medio por las curvas de nivel de 300 y de $200 \mathrm{~m}$.

De este modo contamos ya con todas las variables necesarias para la realización del Test del Vecino más Próximo:

$$
\begin{aligned}
& d=0,3793 \mathrm{Km} . \\
& \mathrm{N}=43 \\
& \mathrm{~A}=39,40 \mathrm{~km}^{2}
\end{aligned}
$$

de donde, mediante la aplicación de la formula anterior, se obtiene un índice $\mathrm{R} \mathrm{n}$ de 0,8279 .

Dado que los valores de $\mathrm{R} n$ varían desde 0 , cuando todos los puntos están agrupados en un solo lugar, hasta 2,15, valor obtenido cuando están tan alejados entre sí como sea posible, formando una distribución regular de tipo hexagonal, y que un valor de 1 indica una distribución aleatoria de los puntos (HAGGETT, 1976), podemos afirmar que, dentro de los límites propuestos, la distribución de asentamientos en el valle del Guadatín tiende hacia una distribución agrupada, con la salvedad de que en este caso ese 
agrupamiento no está en función de un factor puntual sino lineal, lo que implica un patrón de asentamiento con territorios de tendencia rectangular orientados de forma transversal al eje del recurso localizado, en este caso el cauce fluvial (Fig. 3).

Este modelo general se ve en parte distorsionado por varios agrupamientos en tomo a un punto central, como acontece alrededor de los asentamientos 277,188 y 189 . La explicación de este fenómeno no es fácil, aunque pueden enunciarse una serie de factores que contribuyan a su esclarecimiento. En primer lugar, debemos señalar cómo los resultados de toda prospección superficial serán siempre relativos, dependiendo el mayor o menor nivel de fiabilidad de la metodología empleada. Sin embargo, existen factores que escapan a la labor propia del arquélogo pero con los que es preciso contar. Pueden sintetizarse en:

\section{Conservación diferencial de los lugares arqueológicos}

Constituye una variable que normalmente no puede estudiarse de un modo directo, aunque ha sido abordada desde propuestas metodologócias válidas que han tratado de reconstruir la Historia postdeposicional de los yacimientos. Los principales factores que inciden en esta conservación diferencial son:

- Procesos naturales, y en especial la erosión.

- Tipo y grado de utilización antrópica del terreno.

\section{Descubrimiento diferencial de los lugares arqueológicos}

Responde a una variada gama de factores que entroncan con los ya vistos para la conservación diferencial y con los derivados de las prioridades en las líneas de trabajo de los grupos de investigadores. Las distorsiones originadas por esta variable pueden reducirse hasta un nivel aceptable mediante una planificación cientifica y sistemática de la labor de prospección y el auxilio de la fotografía aérea y de técnicas de detección física (sondeos electromagnéticos) o química (análisis de la proporción de fosfatos presente en los suelos).

Atendiendo a lo dicho debemos reconocer cómo, pese al carácter exhaustivo y sistemático de la prospección realizada en el Arroyo Guadatín, en un pasado pudieron existir más yacimientos que los localizados en la actualidad, y del mismo modo en un futuro pueden ponerse al descubierto yacimientos de los que hoy día no se advierte nigún vestigio en superficie. No obstante, los procesos naturales y antrópicos son homogéneos a lo largo de todo el área considerada y la muestra conocida lo suficientemente amplia, por lo que se puede mantener que las tendencias observadas responden a una constante real y no proceden del azar.

Una vez descartada la aleatoriedad de toda prospección como factor relevante en la configuración del poblamiento durante la Fase Colonial u Orientalizante del Bronce 
Final en el Arroyo Guadatín, deberemos buscar en la distribución espacial de los recursos y en la dinámica histórico-cultural de las poblaciones tartésicas una explicación plausible.

Ya hemos indicado que el principal recurso económico de la zona lo constituye una tierra especialmente adecuada para el cultivo de cereales. La aparición en varios de los asentamientos de dientes de hoz con pátina de siega y de molinos barquiformes destinados a triturar los granos de cereal representa una confirmación arqueológica de la vocación cerealista de estas comunidades. Sin embargo la distribución de buenas tierras de cultivo es mucho más amplia que lo que refleja la red de poblamiento, tanto en el Guadatín como en otras zonas de la Campina. En consecuencia debemos buscar otros recursos con una localización espacial más restringida como los principales condicionadores del asentamiento humano.

El abastecimiento de agua puede haber actuado como ese factor catalizador, obligando a que los asentamientos se dispongan a corta distancia, por lo general 100 o $200 \mathrm{~m}$., de los cursos fluviales. Ahora bien como ya analizamos más arriba, la utilización de esas aguas no es factible durante todo el ciclo estacional, debiéndose buscar un aprovisionamiento más estable en los acuíferos subterráneos mediante la perforación de pozos. La distribución actual de los pozos en la zona indica que estos no se localizan de forma exclusiva en las proximidades del Arroyo, y que el nivel freático se alcanza a cotas similares hasta a $2000 \mathrm{~m}$. de distancia de él.

Reducida esta posibilidad deberemos centramos en el aporte estacional de aguas durante las crecidas del Arroyo. Estas, que de acuerdo con el régimen pluviométrico de la Campiña se concentrarían de Noviembre a Enero, supondrfan un volumen adicional de agua que, en parte regulado mediante la construcción de sencillas represas similares a las que aún hoy pueden verse, podria administrarse hasta bien entrada la primavera para cultivos de huerta situados a orillas del cauce fluvial. Por otro lado, los limos arrastrados por las aguas y depositados en los lechos de inundación proporcionarían una capa relativamente fértil sobre la que realizar tales cultivos. Esta complementariedad de cultivos hortícolas y cerealísticos de secano por grupos familiares es una constante advertida, en todo tiempo y lugar, por varios investigadores (BUTZER, 1989; WOLF, 1978).

De cuanto llevamos dicho, se desprende que el patrón de asentamiento a lo largo del Arroyo Guadatín está condicionado por varios factores principales:

- Apropiación de una porción de terreno situada junto al cauce del Arroyo, susceptible de ser transformada en huerta mediante el aprovechamiento estacional de las aguas del mismo.

- Aprovisionamiento regular de agua para el consumo humano y del ganado, asegurado mediante la perforación de pozos.

- Apropiación de un territorio destinado al cultivo de cereales, con una extensión que oscila desde poco más de una veintena de hectáreas (asentamientos 188 y 207) hasta varios centenares. 
Junto a estos condicionantes deberemos considerar el introducido por el carácter de vfa de comunicación entre el valle del Guadalquivir y la Campiña que presenta el valle del Guadatín. En una zona de diff́ciles comunicaciones como la Campiña (LOPEZ ONTIVEROS, 1973), esta proximidad a una ruta es de vital importancia para las comunidades campesinas, constituyendo su única posibilidad de acceder a un flujo de mercancfas de las que no eran productoras, al tiempo que por ella darfan salida a sus excedentes de granos. Sílex para la fabricación de elementos y dientes de hoz, piedras duras para los molinos, sustancias colorantes, sal destinada a la conservación de los alimentos y al consumo del ganado, debieron ser algunas de las materias primas que circularon por esta vía. Lo mismo cabe decir de los objetos de metal (armas y útiles de producción) y/o del mineral necesario para su fabricación, aunque todo ello deb́a estar dirigido hacia los grandes asentamientos del interior de la Campina, pues los núcleos rurales no parecen haber sido consumidores habituales de los mismos, al menos en las cantidades mínimas susceptibles de ser constatadas a partir del registro arqueológico.

Por lo que respecta a objetos manufacturados como la cerámica, debemos distinguir entre las producciones a mano toscas, que muy probablemente se fabricarfan in situ en cada uno de los asentamientos, de las cerámicas a mano cuidadas, en algún caso con decoración brufida, que pudieron manufacturarse en varias unidades productoras más especializadas, aunque ubicadas en el medio rural, y distribuirse en su entomo inmediato, en un radio de varios kms. Por último, un tercer nivel vendría dado por las cerámicas grises y pintadas bícromas fabricadas a torno, que tras una fase inicial en la que constiuirfan un producto exótico importado de los ambientes coloniales fenicios y limitado a los asentamientos de mayor importancia -los integrantes de la red primaria de poblamiento-, pasarfan a fabricarse en alfares indígenas ubicados en centros urbanos o protourbanos, aunque no pueda descartarse la posible instalación de algún pequeño centro productor en el medio rural, como el recientemente excavado de "La Campiña", en Marmolejo (MOLINOS et alii, 1991). De la distribución de estas cerámicas en el medio rural a partir de un momento de mediados del s. VII da buena prueba su presencia en la mayor parte de los yacimientos localizados en el Guadatín (Tabla 1). Esto puede estar en relación con la facilidad de las comunicaciones a lo largo de esta vía y con la relativa prosperidad alcanzada, lo que contrasta con otros asentaientos coetáneos situados en áreas menos favorecidas y en las que estas producciones son menos frecuentes.

Hasta aquf hemos reducido la dimensión temporal de nuestro estudio a su aspecto sincrónico, dentro de un intervalo aproximado de siglo y medio. Sin embargo el proceso de ocupación del territorio, dentro de un fenómeno que podríamos calificar como de colonización no debió ser estático, sino dinámico, siendo el panorama que acabamos de presentar, con toda probabilidad, el resultado final del mismo. Por desgracia, un intento de estudio diacrónico choca con las limitaciones impuestas por la naturaleza de la Jocumentacion susceptible de proporcionarnos cronologia, esto es, la cerámica. En el 
estado actual de la investigación, las posibilidades de precisar la cronología de un fragmento de cerámica tartésica fabricada a mano no pasan de generalizaciones del tipo de comienzos del s. VIII, mediados del VII, etc. Lo mismo cabe decir, con márgenes algo menores, para las cerámicas fabricadas a tomo, ya sean grises, de engobe rojo o pintadas. En consecuencia, intentar fijar etapas dentro de un intervalo histórico de siglo y medio a partir de una prospección superficial y sin la excavación de un número estadísticamente significativo de yacimientos no deja de resultar utópico.

Aunque nos veamos obligados a permanecer dentro de la esfera de lo que la escuela de Braudel denominaría estructural, no por ello debemos renunciar a reconstruir, con carácter de hipótesis por supusto, lo que pudo ser el proceso de ocupación de este territorio. La usencia de cerámicas decoradas a la almagra, pintadas con motivos geométricos tipo Guadalquivir I y con incrustación de botones metálicos, todas ellas presentes en yacimientos situados en el Valle del Guadalquivir y en otras áreas próximas (MURILLO, 1991) apuntan a una no-ocupación del Guadatín durante el Bronce Final Precolonial.

Tras unos remotos precedentes remontables al Calcolítico, los primeros asentamientos parecen datar de un momento de la primera mitad del s. VII, a juzgar por la cronología proporcionada por varios fragmentos de cerámica gris y por los perfiles evolucionados de las cazuelas de carena alta fabricadas a mano. De acuerdo con modelos de colonización de espacios similares al nuestro (HAGGETT, 1976; HODDER et ORTON, 1976). en tan sólo cinco generaciones -aproximadamente los 150 años en que nos venimos moviendo- se pudo haber alcanzado la ocupación del territorio partiendo de un número reducido de núcleos pioneros. La delimitación del número de estas comunidades primitivas dependerá del crecimiento vegetativo experiemntado por una sociedad preindustrial y rural como la que nos ocupa (CIPOLLA, 1981).

Si consideramos una alta natalidad que se vería compensada por una mortalidad infantil también elevada, podría resultar aceptable una cifra entre cinco y seis hijos por pareja que alcanzaran la edad adulta. Si prescindimos de controles culturales que alteraran la proporción normal entre los sexos (HARRIS, 1988) y admitimos un sistema de descendencia patriarcal como el más probable entre las comunidades tartésicas, obtendríamos entre 2 y 3 hijos varones por pareja y generación. De ellos uno quedaría al cuidado de la explotación madre, en tanto que el o los restantes deberían roturar un nuevo territorio. De este modo, serían necesarios entre 2 y 8 asentamientos-matriz para alcanzar el resultdo final que muestra la Fig. 3.

A juzgar por el material cerámico, este proceso estaba concluido hacia mediados del s. VI, sin que debamos interpretar la diferencia en el tamaño de los territorios teóricos (Fig. 3) como prueba de una colonización incompleta, pues ésta podría explicarse en función de unos recursos críticos más limitados que deben compensarse con una mayor extensión. A este respecto, la localización de estos territorios más amplios en el curso medio-alto del Guadatín podría resultar significativa. La relación que este hecho pudiera 
tener con la crisis del mundo tartésico que se advierte por las mismas fechas (ESCACENA, 1987) es sugerente, aunque deberemos contar con más elementos de juicio para pronunciaros, por lo que la respuesta a esta cuestión deberá aplazarse a un próximo trabajo.

\section{Bibliografia}

BUTZER, K.W. (1989); Arqueología. Una ecología del hombre, Barcelona. CEBAC (1971); Estudio agrobiológico de la provincia de Córdoba, Madrid. CIPOLLA, C.M. (1981); Historia económica de la Europa preindustrial, Madrid. ESCACENA, J.L. (1987); "El poblamiento ibérico en el Alto Guadalquivir", Iberos, pp. 273-298.

GILMAN, A. et alii (1985); Land-use prehistory in south-east Spain, Lodon. HAGGETT, P. (1976); Análisis locacional en la Geografia Humana, Barcelona. HARRIS, M. (1988); Introducción a la Antropología general, Madrid. HODDER, I. et ORTON, C. (1976); Spatial analysis in archaeology, London. LOPEZ ONTIVEROS, A. (1973); " Rasgos geomorfologicos de la Capiña de Córdoba”, Estudios Geográficos, 130, pp. 33-94.

ORTON, C. (1988); Matemáticas para arqueólogos, Madrid.

MOLINOS, M.; SERRANO, J.L.; COBA, B. (1991); “'Excavaciones arqueológicas en el asentamiento de La Campiña. Marmolejo, Jaén”, $A A A^{\prime}$ 89, VOL. III, pp. 197-203. MURILLO, J.F. (1991); "Análisis del poblamiento durante el Bronce Final y el Orientalizante en la Cuenca Media del Guadalquivir". Tesis Doctoral, Universidad de Córdoba (inédita).

ROSA, D. de la et MOREIRA, J.M. (1987); Evaluación ecológica de recursos naturales de Andalucia, Sevilla, 1987.

WOLF, E.R. (1978); Los campesinos, Barcelona. 


\begin{tabular}{|c|c|c|c|c|c|c|c|c|c|c|}
\hline & & & C. Mano & C.M. Dec. & c. Torno & P.Bicr. & P.Mon. & Gris & D. $\mathrm{Hoz}$ & $\mathrm{Mol}$. \\
\hline 120 & Algarrobilion II & Cordoba & 1 & 0 & 1 & 1 & 1 & 0 & 0 & 0 \\
\hline 131 & Cardenas I & Cordoba & 1 & 0 & 1 & 1 & 1 & 1 & 0 & 1 \\
\hline 132 & Cardenas it & Cordoba & 1 & 0 & 1 & 1 & 1 & 1 & 0 & 1 \\
\hline 145 & Corti jo Redonde & Corcloba & 1 & 1 & 1 & 0 & 1 & 0 & 0 & 0 \\
\hline 146 & Corti jo de Abolafia & Cordoba & 1 & 0 & 1 & 1 & 1 & 0 & 0 & 0 \\
\hline 153 & Corti jo de Rubililas Bajas & Cordoba & 1 & 1 & 1 & 0 & 0 & 1 & 1 & 0 \\
\hline 155 & Corti jo de Tafarrilia & Corchba & 1 & 0 & 1 & 1 & 1 & 1 & 0 & 0 \\
\hline 162 & Cort 1 jo de las Hazuelas & Corctoba & 1 & 0 & 1 & 0 & 1 & 0 & 0 & 2 \\
\hline 164 & Cortl jo de loe Algarrobillos & Cordoba & 1 & 0 & 1 & 0 & 1 & 0 & 0 & 1 \\
\hline 165 & Cortil jo de loe Harineros & Cordoba & 1 & 1 & 1 & 1 & 1 & 1 & 1 & 1 \\
\hline 172 & Corti jo del Jaro Bajo V & Corcoba & 1 & 0 & 1 & 0 & 1 & 0 & 0 & 0 \\
\hline 175 & El Caracol & Corcloba & 1 & 0 & 1 & 1 & 1 & 1 & 0 & 1 \\
\hline 178 & Extraviados I & Corcioba & 1 & 0 & 1 & 1 & 1 & 0 & 0 & 1 \\
\hline 179 & Extraviados 11 & Corcoba & 1 & 0 & 1 & 1 & 1 & 1 & 0 & 0 \\
\hline 184 & Haza Ancha & Corcoba & 1 & 0 & 1 & 1 & 1 & 1 & 0 & 0 \\
\hline 185 & Hazuelas Bajan & Cordoba & 1 & 0 & 1 & 1 & 1 & 0 & 0 & 0 \\
\hline 186 & Jaro Bajo 1 & Corcoba & 1 & 0 & 1 & 1 & 1 & 1 & 0 & 0 \\
\hline 187 & Jaro Bajo It & Cordoba & 1 & 0 & 1 & 1 & 1 & 1 & 0 & 0 \\
\hline $1 \otimes \theta$ & Jaro Ba jo III & Cordoba & 1 & 0 & 1 & 1 & 1 & 0 & 0 & 0 \\
\hline 189 & Jaro Bajo IV & Cordoba & 1 & 0 & 0 & 0 & 0 & 0 & 0 & 1 \\
\hline 192 & María Apariclo & Corcoba & 1 & 0 & 1 & 1 & 1 & 1 & 0 & 0 \\
\hline 195 & Recondo & Cordoba & 1 & 0 & 1 & 1 & 1 & 1 & 0 & 1 \\
\hline 196 & Rub111as Altas & Cordoba & 1 & 0 & 1 & 1 & 1 & 0 & 0 & 1 \\
\hline 199 & Tafarra & Cordoba & 1 & 0 & 1 & 1 & 1 & 1 & 0 & 0 \\
\hline 200 & Torrecillas I & Cordoba & 1 & 0 & 1 & 1 & 1 & 1 & 0 & 0 \\
\hline 201 & Torrecillas II & Cordoba & 1 & 0 & 1 & 1 & 1 & 0 & 0 & 0 \\
\hline 202 & Torrecillas III & Cordoba & 1 & 0 & 1 & 1 & 1 & 0 & 0 & 1 \\
\hline 204 & Traparo I & Cordoba & 1 & 0 & 1 & 1 & 1 & 1 & 0 & 1 \\
\hline 205 & Trapero II & Corcoba & 1 & 0 & 1 & 1 & 1 & 0 & 0 & 1 \\
\hline 207 & Valsequill o I & Cordoba & 1 & 0 & 1 & 1 & 1 & 0 & 0 & 0 \\
\hline 208 & Valsequillo II & Cordoba & 1 & 0 & 1 & 1 & 1 & 0 & 0 & 0 \\
\hline 275 & Cortijo de Guadatín & Villafranca & 1 & 0 & 1 & 0 & 0 & 1 & 0 & 1 \\
\hline 276 & Qumbres Bajas & Villafranca & 1 & 0 & 1 & 1 & 1 & 0 & 0 & 1 \\
\hline 277 & Guadatin Alto I & Villafranca & 1 & 0 & 1 & 1 & 1 & 1 & 0 & 1 \\
\hline 278 & Guadatín Alto II & Villafranca & 1 & 1 & 0 & 0 & 0 & 0 & 0 & 0 \\
\hline 279 & Quadatín Bajo I & Villafranca & 1 & 0 & 1 & 1 & 1 & 1 & 0 & 0 \\
\hline 280 & Guadatín Bajo II & Villafranca & 1 & 0 & 1 & 1 & 1 & 0 & 0 & 0 \\
\hline 281 & Guadatín Medio I & Villafranca & 1 & 0 & 1 & 1 & 1 & 1 & 0 & 1 \\
\hline 282 & Guadatín Medio II & Uillafranca & 1 & 0 & 1 & 1 & 1 & 1 & 0 & 0 \\
\hline 283 & Guadatín Madio III & Villafranca & 1 & 0 & 1 & 1 & 1 & 1 & 0 & 1 \\
\hline 284 & San Franci sco & Villafranca & 1 & 1 & 1 & 1 & 1 & 1 & 0 & 0 \\
\hline 285 & Val sequillo III & Villafranca & 1 & 0 & 1 & 1 & 1 & 1 & 0 & 1 \\
\hline 286 & Dorti jo del Eucalipto & Villafranca & 1 & 1 & 1 & 0 & 0 & 0 & 0 & 0 \\
\hline
\end{tabular}

Nota: 0 equivale a ausencia del 1 tem.

1 equivale a presencia del iten. 


\begin{tabular}{|c|c|c|}
\hline № YACIMIENTO (*) & VECINO MAS PROXIMO & DISTANCIA (Metros) \\
\hline 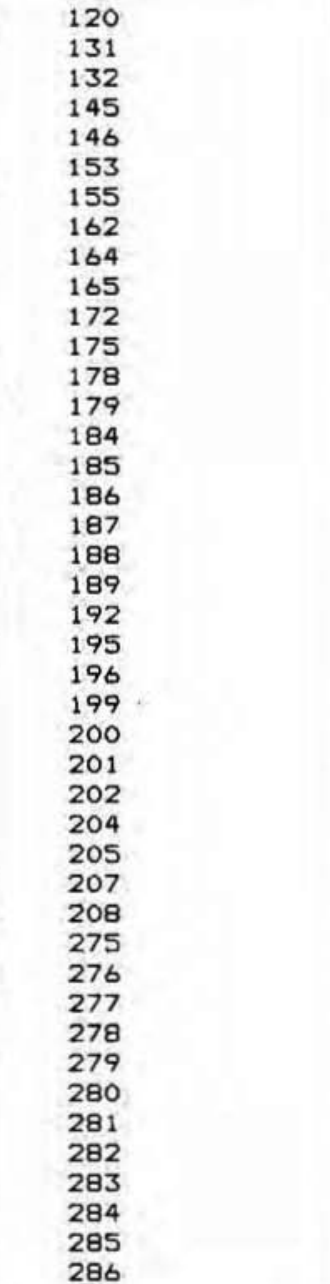 & $\begin{array}{l}164 \\
187 \\
131 \\
189 \\
155 \\
200 \\
199 \\
185 \\
120 \\
188 \\
189 \\
184 \\
179 \\
178 \\
207 \\
205 \\
189 \\
188 \\
187 \\
186 \\
146 \\
164 \\
199 \\
155 \\
201 \\
202 \\
201 \\
205 \\
204 \\
208 \\
207 \\
279 \\
280 \\
278 \\
285 \\
283 \\
275 \\
282 \\
281 \\
279 \\
276 \\
278 \\
277\end{array}$ & $\begin{array}{l}310 \\
450 \\
700 \\
340 \\
415 \\
710 \\
400 \\
425 \\
310 \\
220 \\
580 \\
275 \\
390 \\
390 \\
250 \\
400 \\
320 \\
210 \\
210 \\
320 \\
1650 \\
450 \\
700 \\
400 \\
160 \\
140 \\
140 \\
350 \\
350 \\
300 \\
300 \\
180 \\
625 \\
275 \\
250 \\
150 \\
250 \\
160 \\
160 \\
150 \\
950 \\
280 \\
315\end{array}$ \\
\hline
\end{tabular}

(*)La numeración corresponde a la general adoptada en MURILLO (1991)

TABLA 2 


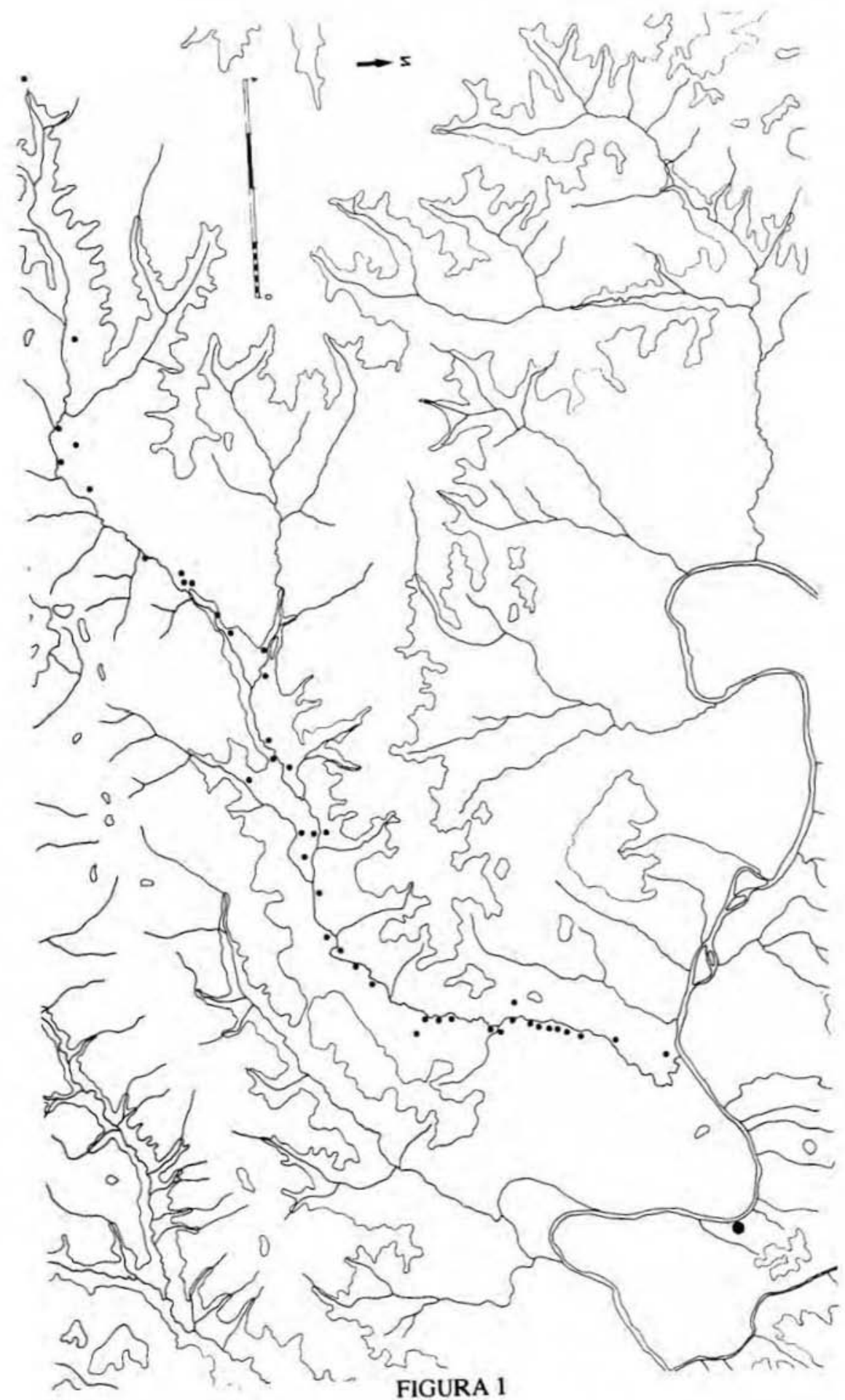

--- Grupo de investigación P.A.I. HUM 236 | http://www.arqueocordoba.com/publ/anales.htm --- 


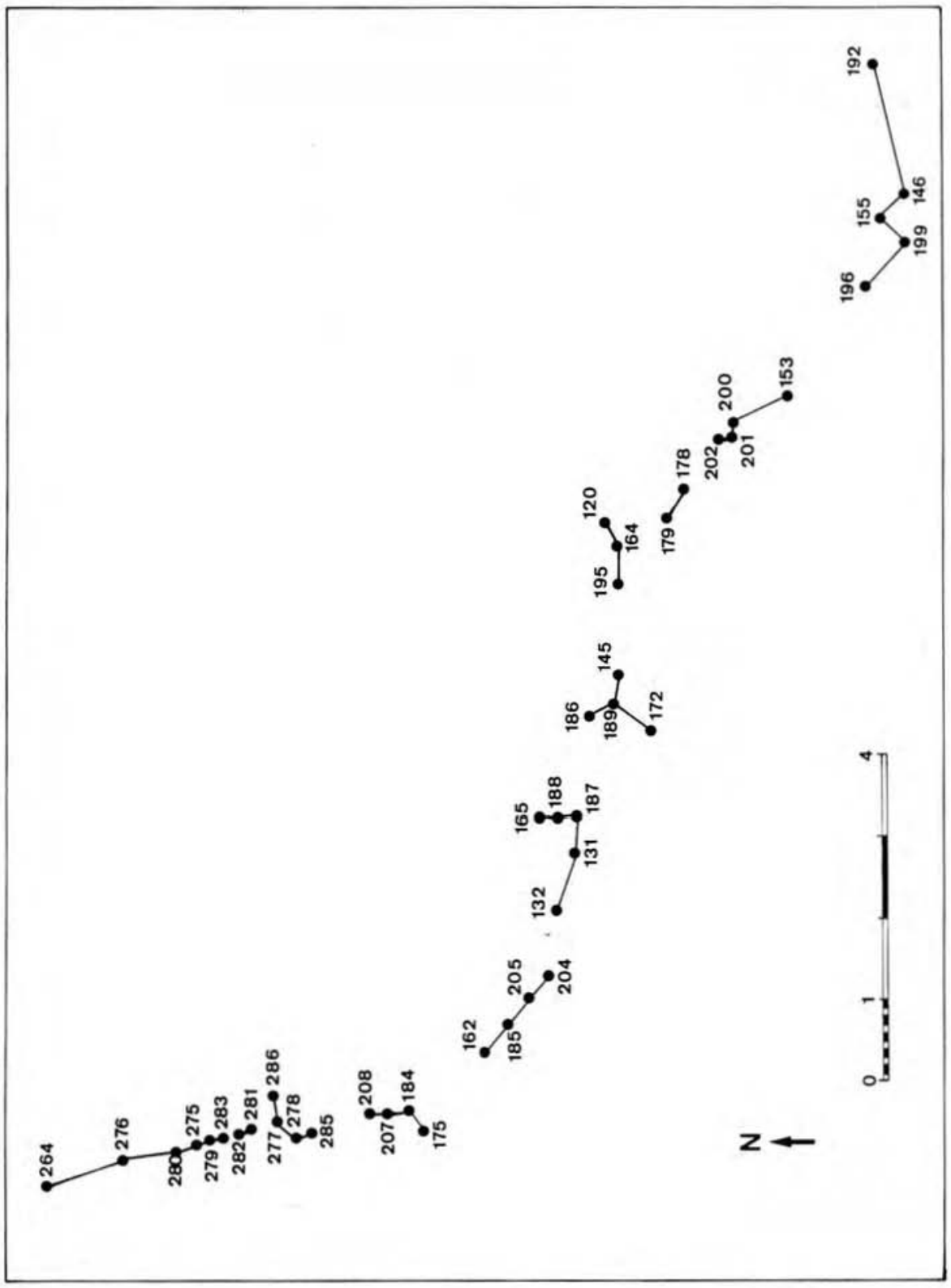

FIGURA 2 


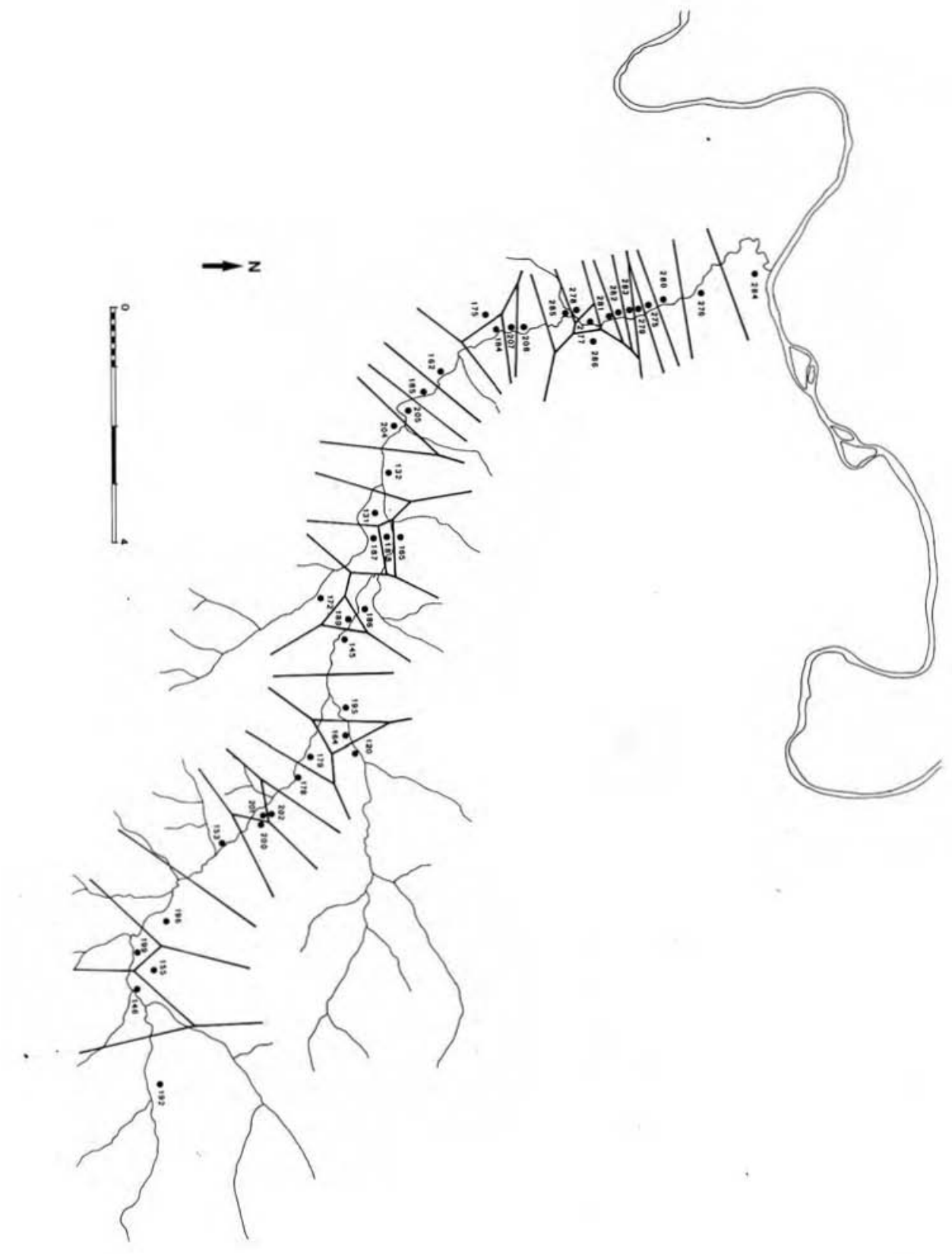

FIGURA 3 